\title{
Iterative methods for split variational inclusion and fixed point problem of nonexpansive semigroup in Hilbert spaces
}

\section{Dao-Jun Wen* and Yi-An Chen}

"Correspondence:
daojunwen@163.com
College of Mathematics and
Statistics, Chongqing Technology
and Business University, Chongqing,
400067, China

400067, China

\begin{abstract}
In this paper, we introduce a general iterative method for a split variational inclusion and nonexpansive semigroups in Hilbert spaces. We also prove that the sequences generated by the proposed algorithm converge strongly to a common element of the set of solutions of a split variational inclusion and the set of common fixed points of one-parameter nonexpansive semigroups, which also solves a class of variational inequalities as an optimality condition for a minimization problem. Moreover, a numerical example is given, to illustrate our methods and results, which may be viewed as a refinement and improvement of the previously known results announced by many other authors.
\end{abstract}

MSC: 47H09;47H10;47J22

Keywords: split variational inclusion; nonexpansive semigroup; fixed point; averaged mapping; general iterative method

\section{Introduction}

Let $H_{1}$ and $H_{2}$ be real Hilbert spaces with inner product $\langle\cdot, \cdot\rangle$ and norm $\|\cdot\|$, respectively. Recall that a mapping $T: H_{1} \rightarrow H_{1}$ is called nonexpansive if

$$
\|T x-T y\| \leq\|x-y\|, \quad \forall x, y \in H_{1} .
$$

A one-parameter family $\mathscr{T}:=\{T(s): 0 \leq s<\infty\}$ is said to be a nonexpansive semigroup on $H_{1}$ if the following conditions are satisfied:

(1) $T(0) x=x$ for all $x \in H_{1}$;

(2) $T(s+t)=T(s) T(t)$ for all $s, t \geq 0$;

(3) $\|T(s) x-T(s) y\| \leq\|x-y\|$, for all $x, y \in H_{1}$ and $s>0$;

(4) for each $x \in H_{1}$, the mapping $s \mapsto T(s) x$ is continuous.

Denote by $\operatorname{Fix}(\mathscr{T})$ the common fixed point set of the semigroup $\mathscr{T}$, i.e., $\operatorname{Fix}(\mathscr{T}):=\{x \in$ $\left.H_{1}: T(s) x=x, \forall s>0\right\}$. It is well known that $\operatorname{Fix}(\mathscr{T})$ is closed and convex (see Lemma 1 in Browder [1]).

Recently, the fixed point problem of nonexpansive mappings and its iterative methods have become an attractive subject, and various algorithms have been developed for solving variational inequalities and equilibrium problems; see $[2-8]$ and the references therein. In 
2006, Marino and Xu [2] introduced the following general iterative methods to approximate a fixed point of a nonexpansive mapping:

$$
x_{n+1}=\alpha_{n} \gamma f\left(x_{n}\right)+\left(I-\alpha_{n} B\right) T x_{n},
$$

where $\alpha_{n} \in[0,1]$ satisfies certain conditions, $f$ is a contraction of $H_{1}$ into itself, and $B$ is a strongly positive bounded linear operator on $H_{1}$. Moreover, they prove that $\left\{x_{n}\right\}$ converges strongly to $x^{*} \in \operatorname{Fix}(T)$, the unique solution of the following variational inequality:

$$
\left\langle(B-\gamma f) x^{*}, x^{*}-w\right\rangle \leq 0, \quad \forall w \in \operatorname{Fix}(T)
$$

which is also the optimality condition of the minimization problem. Thereafter, Li et al. [3] and Cianciaruso et al. [4] modified the general iterative method (1.1) to the case of nonexpansive semigroups and equilibrium problems. To obtain a mean ergodic theorem of nonexpansive mappings, Shehu [5] proposed an iterative method for nonexpansive semigroups, variational inclusions, and generalized equilibrium problems.

Recall also that a multi-valued mapping $M: H_{1} \rightarrow 2^{H_{1}}$ is called monotone if, for all $x, y \in$ $H_{1}, u \in M x$ and $v \in M y$ such that

$$
\langle x-y, u-v\rangle \geq 0
$$

A monotone mapping $M$ is maximal if the $\operatorname{Graph}(M)$ is not properly contained in the graph of any other monotone mapping. It is well known that a monotone mapping $M$ is maximal if and only if for $(x, u) \in H_{1} \times H_{1},\langle x-y, u-v\rangle \geq 0$ for every $(y, v) \in \operatorname{Graph}(M)$ implies that $u \in M x$.

Let $M: H_{1} \rightarrow 2^{H_{1}}$ be a multi-valued maximal monotone mapping. Then the resolvent mapping $J_{\lambda}^{M}: H_{1} \rightarrow H_{1}$ associated with $M$ is defined by

$$
J_{\lambda}^{M}(x):=(I+\lambda M)^{-1}(x), \quad \forall x \in H_{1}
$$

for some $\lambda>0$, where $I$ stands for the identity operator on $H_{1}$. Note that for all $\lambda>0$ the resolvent operator $J_{\lambda}^{M}$ is single-valued, nonexpansive, and firmly nonexpansive.

In 2011, Moudafi [9] introduced the following split monotone variational inclusion problem: Find $x^{*} \in H_{1}$ such that

$$
\left\{\begin{array}{l}
0 \in f_{1}\left(x^{*}\right)+B_{1}\left(x^{*}\right), \\
y^{*}=A x^{*} \in H_{2}: \quad 0 \in f_{2}\left(y^{*}\right)+B_{2}\left(y^{*}\right),
\end{array}\right.
$$

where $B_{1}: H_{1} \rightarrow 2^{H_{1}}$ and $B_{2}: H_{2} \rightarrow 2^{H_{2}}$ are multi-valued maximal monotone mappings. The split monotone variational inclusion problem (1.2) includes as special cases: the split common fixed point problem, the split variational inequality problem, the split zero problem, and the split feasibility problem, which have already been studied and used in practice as a model in intensity-modulated radiation therapy treatment planning, see e.g. [10-12]. This formalism is also at the core of the modeling of many inverse problems arising for phase retrieval and other real-world problems; for instance, in sensor networks in computerized tomography and data compression; see $[13,14]$ and the references therein. 
If $f_{1} \equiv 0$ and $f_{2} \equiv 0$, then problem (1.2) reduces to the following split variational inclusion problem: Find $x^{*} \in H_{1}$ such that

$$
\left\{\begin{array}{l}
0 \in B_{1}\left(x^{*}\right), \\
y^{*}=A x^{*} \in H_{2}: \quad 0 \in B_{2}\left(y^{*}\right),
\end{array}\right.
$$

which constitutes a pair of variational inclusion problems connected with a bounded linear operator $A$ in two different Hilbert spaces $H_{1}$ and $H_{2}$. The solution set of problem (1.3) is denoted by $\mathscr{Z}=\left\{x^{*} \in H_{1}: 0 \in B_{1}\left(x^{*}\right), y^{*}=A x^{*} \in H_{2}: 0 \in B_{2}\left(y^{*}\right)\right\}$.

Very recently, Byrne et al. [15] studied the weak and strong convergence of the following iterative method for problem (1.3): For given $x_{0} \in H_{1}$ and $\lambda>0$, compute iterative sequence $\left\{x_{n}\right\}$ generated by the following scheme:

$$
x_{n+1}=J_{\lambda}^{B_{1}}\left[x_{n}+\epsilon A^{*}\left(J_{\lambda}^{B_{2}}-I\right) A x_{n}\right] .
$$

In 2013, Kazmi and Rizvi [16] modified scheme (1.3) to the case of a split variational inclusion and the fixed point problem of a nonexpansive mapping. To be more precise, they proved the following strong convergence theorem.

Theorem KR Let $H_{1}$ and $H_{2}$ be two real Hilbert spaces and $A: H_{1} \rightarrow H_{2}$ be a bounded linear operator. Let $f: H_{1} \rightarrow H_{1}$ be a contraction mapping with constant $\rho \in(0,1)$ and $T: H_{1} \rightarrow H_{1}$ be a nonexpansive mapping such that $\Omega=\operatorname{Fix}(T) \cap \mathscr{Z} \neq \emptyset$. For a given $x_{0} \in H_{1}$ arbitrarily, let the iterative sequences $\left\{u_{n}\right\}$ and $\left\{x_{n}\right\}$ be generated by

$$
\left\{\begin{array}{l}
u_{n}=J_{\lambda}^{B_{1}}\left[x_{n}+\epsilon A^{*}\left(J_{\lambda}^{B_{2}}-I\right) A x_{n}\right], \\
x_{n+1}=\alpha_{n} f\left(x_{n}\right)+\left(1-\alpha_{n}\right) T u_{n},
\end{array}\right.
$$

where $\lambda>0$ and $\epsilon \in(0,1 / L), L$ is the spectral radius of the operator $A^{*} A$, and $A^{*}$ is the adjoint of $A ;\left\{\alpha_{n}\right\}$ is a sequence in $(0,1)$ such that $\lim _{n \rightarrow \infty} \alpha_{n}=0, \sum_{n=1}^{\infty} \alpha_{n}=\infty$, and $\sum_{n=1}^{\infty}\left|\alpha_{n}-\alpha_{n-1}\right|<\infty$. Then the sequences $\left\{u_{n}\right\}$ and $\left\{x_{n}\right\}$ both converge strongly to $z \in \Omega$, where $z=P_{\Omega} f(z)$.

Inspired and motivated by research going on in this area, we introduce a modified general iterative method for a split variational inclusion and nonexpansive semigroups, which is defined in the following way:

$$
x_{n+1}=\alpha_{n} \gamma f\left(x_{n}\right)+\left(I-\alpha_{n} B\right) \frac{1}{s_{n}} \int_{0}^{s_{n}} T(s) J_{\lambda}^{B_{1}}\left[x_{n}+\epsilon A^{*}\left(J_{\lambda}^{B_{2}}-I\right) A x_{n}\right] d s,
$$

where $\gamma \in[0,1]$ and $\alpha_{n}, \beta_{n} \in[0,1], B$ is a strongly positive bounded linear operator on $H_{1}$. Note that, if $\gamma=1, B=I$, and $T(s)=T$, a nonexpansive mapping, scheme (1.6) reduces to the approximate method (1.5), which is mainly due to Byrne et al. [15] and has been applied by Kazmi and Rizvi [16].

Our purpose is not only to modify the general iterative method (1.5) to the case of a split variational inclusion and nonexpansive semigroups from a nonexpansive mapping, but also to prove that the sequences generated by the proposed algorithm converge strongly to a common element of the set of solutions of a split variational inclusion and the set of common fixed points of one-parameter nonexpansive semigroups, which also solves a class of variational inequalities as an optimality condition for a minimization problem. 
Moreover, a numerical example is given to illustrate our algorithm and our results, which improve and extend the corresponding results of $[2,4,5,15,16]$ and many others.

\section{Preliminaries}

Let $C$ be a nonempty, closed, and convex subset of a real Hilbert space $H_{1}$. For every point $x \in H_{1}$, there exists a unique nearest point in $C$, denoted by $P_{C}$, such that

$$
\left\|x-P_{C} x\right\| \leq\|x-y\|, \quad \forall y \in C .
$$

Then $P_{C}$ is called the metric projection of $H_{1}$ onto $C$. It is well known that $P_{C}$ is a nonexpansive mapping and the following inequality holds:

$$
\langle x-u, y-u\rangle \leq 0, \quad \forall y \in C,
$$

if and only if $u=P_{C} x$ for given $x \in H_{1}$ and $u \in C$.

Recall that a mapping $f: H_{1} \rightarrow H_{1}$ is a contraction, if there exists a constant $\rho \in(0,1)$ such that

$$
\|f(x)-f(y)\| \leq \rho\|x-y\|, \quad \forall x, y \in H_{1}
$$

Throughout the rest of this paper, we always assume that $B$ is strongly positive; that is, there is a constant $\bar{\gamma}>0$ such that

$$
\langle B x, x\rangle \geq \bar{\gamma}\|x\|^{2}, \quad \forall x \in H_{1} .
$$

A mapping $S: H_{1} \rightarrow H_{1}$ is said to be averaged if and only if it can be written as the average of the identity mapping and a nonexpansive mapping, i.e., $S:=(1-\alpha) I+\alpha T$ where $\alpha \in(0,1)$ and $T: H_{1} \rightarrow H_{1}$ is nonexpansive and $I$ is the identity operator on $H_{1}$. We note that averaged mappings are nonexpansive. Further, firmly nonexpansive mappings (in particular, projections on nonempty, closed, and convex subsets and resolvent operators of maximal monotone operators) are averaged.

In order to prove our main results, we need the following lemmas and propositions.

Lemma 2.1 Let $H_{1}$ be a real Hilbert space. The following well-known results hold:

(i) $\|x+y\|^{2} \leq\|x\|^{2}+2\langle y,(x+y)\rangle, \forall x, y \in H_{1}$;

(ii) $\|t x+(1-t) y\|^{2}=t\|x\|^{2}+(1-t)\|y\|^{2}-t(1-t)\|x-y\|^{2}, t \in[0,1], \forall x, y \in H_{1}$.

Lemma 2.2 [4, 17] Let D be a nonempty, bounded, closed, and convex subset of a real Hilbert space $H$ and let $\mathscr{T}:=\{T(s): 0 \leq s<\infty\}$ a nonexpansive semigroup on $D$, then for any $u \geq 0$,

$$
\lim _{t \rightarrow \infty} \sup _{x \in D}\left\|\frac{1}{t} \int_{0}^{t} T(s) x d s-T(u) \frac{1}{t} \int_{0}^{t} T(s) x d s\right\|=0 .
$$

Lemma 2.3 $[5,18]$ Let $S: H_{1} \rightarrow H_{1}$ be averaged and $T: H_{1} \rightarrow H_{1}$ be nonexpansive; we have:

(i) $W=(1-\alpha) S+\alpha T$ is averaged, where $\alpha \in(0,1)$.

(ii) The composite of finitely many averaged mappings is averaged. 
Lemma 2.4 [16] The split variational inclusion problem (1.5) is equivalent to finding $x^{*} \in$ $H_{1}$ such that $y^{*}=A x^{*} \in H_{2}: x^{*}=J_{\lambda}^{B_{1}}\left(x^{*}\right)$ and $y^{*}=J_{\lambda}^{B_{2}}\left(y^{*}\right)$ for some $\lambda>0$.

Lemma 2.5 [2] Let B be a strongly positive linear bounded operator on a Hilbert space $H_{1}$ with a coefficient $\bar{\gamma}>0$ and $0<\varrho<\|B\|^{-1}$. Then $\|I-\varrho B\| \leq 1-\varrho \bar{\gamma}$.

Lemma 2.6 [2] Let $C$ be a nonempty, closed, and convex subset of a Hilbert space $H_{1}$. Assume that $f: C \rightarrow C$ is a contraction with a coefficient $\rho \in(0,1)$ and $B$ is a strongly positive linear bounded operator with a coefficient $\bar{\gamma}>0$. Then, for $0<\gamma<\bar{\gamma} / \rho$,

$$
\langle x-y,(B-\gamma f) x-(B-\gamma f) y\rangle \geq(\bar{\gamma}-\gamma \rho)\|x-y\|^{2}, \quad \forall x, y \in H_{1} .
$$

That is, $B-\gamma f$ is strongly monotone with coefficient $\bar{\gamma}-\gamma \rho$.

Lemma 2.7 [19] Let $\left\{a_{n}\right\}_{n=1}^{\infty}$ be a sequence of nonnegative real numbers such that

$$
a_{n+1} \leq\left(1-\gamma_{n}\right) a_{n}+\gamma_{n} b_{n}+\sigma_{n}
$$

where $\left\{\gamma_{n}\right\}_{n=1}^{\infty} \subset(0,1)$ and $\left\{b_{n}\right\}_{n=1}^{\infty},\left\{\sigma_{n}\right\}_{n=1}^{\infty}$ are sequences in $\mathbb{R}$ such that

(i) $\lim _{n \rightarrow \infty} \gamma_{n}=0$ and $\sum_{n=1}^{\infty} \gamma_{n}=\infty$;

(ii) $\limsup _{n \rightarrow \infty} b_{n} \leq 0$;

(iii) $\sigma_{n} \geq 0$ and $\sum_{n=1}^{\infty} \sigma_{n}<\infty$.

Then $\lim _{n \rightarrow \infty} a_{n}=0$.

\section{Main results}

Theorem 3.1 Let $H_{1}$ and $H_{2}$ be two real Hilbert spaces, let $A: H_{1} \rightarrow H_{2}$ be a bounded linear operator and $B$ be a strongly positive bounded linear operator on $H_{1}$ with constant $\bar{\gamma}>0$. Let $B_{1}: H_{1} \rightarrow 2^{H_{1}}, B_{2}: H_{2} \rightarrow 2^{H_{1}}$ be maximal monotone mappings and $\mathscr{T}:=\{T(s):$ $0 \leq s<\infty\}$ be a one-parameter nonexpansive semigroup on $H_{1}$ such that $\operatorname{Fix}(\mathscr{T}) \cap \mathscr{Z} \neq \emptyset$. Assume that $f: H_{1} \rightarrow H_{1}$ is a contraction mapping with constant $\rho \in(0,1)$. For any $\alpha \in$ $(0,1)$, define the mapping $\Phi$ on $H_{1}$ by

$$
\Phi(x)=\alpha \gamma f(x)+(I-\alpha B) \frac{1}{t} \int_{0}^{t} T(s) J_{\lambda}^{B_{1}}\left[x+\epsilon A^{*}\left(J_{\lambda}^{B_{2}}-I\right) A x\right] d s,
$$

where $t>0, \gamma \in\left(0, \frac{\bar{\gamma}}{\rho}\right)$, and $\epsilon \in\left(0, \frac{1}{L}\right), L$ is the spectral radius of the operator $A^{*} A$, and $A^{*}$ is the adjoint of $A$. Then the mapping $\Phi$ is a contraction and has a unique fixed point.

Proof Since $J_{\lambda}^{B_{1}}$ and $J_{\lambda}^{B_{2}}$ are firmly nonexpansive, they are averaged. For $\epsilon \in(0,1 / L)$, the mapping $I+\epsilon A^{*}\left(J_{\lambda}^{B_{2}}-I\right) A$ is averaged; see e.g. [9]. It follows from Lemma 2.3(ii) that the mapping $J_{\lambda}^{B_{1}}\left(I+\epsilon A^{*}\left(J_{\lambda}^{B_{2}}-I\right) A\right)$ is averaged and hence nonexpansive. By Lemma 2.5 , for any $x, y \in H_{1}$, we have

$$
\begin{aligned}
\|\Phi(x)-\Phi(y)\|= & \| \alpha \gamma f(x)+(I-\alpha B) \frac{1}{t} \int_{0}^{t} T(s) J_{\lambda}^{B_{1}}\left[x+\epsilon A^{*}\left(J_{\lambda}^{B_{2}}-I\right) A x\right] d s \\
& -\alpha \gamma f(y)-(I-\alpha B) \frac{1}{t} \int_{0}^{t} T(s) J_{\lambda}^{B_{1}}\left[y+\epsilon A^{*}\left(J_{\lambda}^{B_{2}}-I\right) A y\right] d s \|
\end{aligned}
$$




$$
\begin{aligned}
\leq & \alpha \gamma\|f(x)-f(y)\|+(1-\alpha \bar{\gamma}) \| J_{\lambda}^{B_{1}}\left[x+\epsilon A^{*}\left(J_{\lambda}^{B_{2}}-I\right) A x\right] \\
& -J_{\lambda}^{B_{1}}\left[y+\epsilon A^{*}\left(J_{\lambda}^{B_{2}}-I\right) A y\right] \| \\
\leq & \alpha \gamma \rho\|x-y\|+(1-\alpha \bar{\gamma})\|x-y\| \\
= & {[1-\alpha(\bar{\gamma}-\gamma \rho)]\|x-y\| . }
\end{aligned}
$$

It follows from $\gamma \in\left(0, \frac{\bar{\gamma}}{\rho}\right)$ that $\Phi$ is a contraction mapping. Therefore, by the Banach contraction principle, $\Phi(x)$ has a unique fixed point $x_{\alpha}$, that is,

$$
\left.x_{\alpha}=\alpha \gamma f\left(x_{\alpha}\right)+(I-\alpha B) \frac{1}{t} \int_{0}^{t} T(s)\right)_{\lambda}^{B_{1}}\left[x_{\alpha}+\epsilon A^{*}\left(J_{\lambda}^{B_{2}}-I\right) A x_{\alpha}\right] d s .
$$

Theorem 3.2 Let $H_{1}$ and $H_{2}$ be two real Hilbert spaces, let $A: H_{1} \rightarrow H_{2}$ be a bounded linear operator and $B$ be a strongly positive bounded linear operator on $H_{1}$ with constant $\bar{\gamma}>0$. Let $B_{1}: H_{1} \rightarrow 2^{H_{1}}, B_{2}: H_{2} \rightarrow 2^{H_{1}}$ be maximal monotone mappings and $\mathscr{T}:=\{T(s): 0 \leq s<\infty\}$ be a one-parameter nonexpansive semigroup on $H_{1}$ such that $\Omega=\operatorname{Fix}(\mathscr{T}) \cap \mathscr{Z} \neq \emptyset$. Assume that $f: H_{1} \rightarrow H_{1}$ is a contraction mapping with constant $\rho \in(0,1), L$ is the spectral radius of the operator $A^{*} A$, and $A^{*}$ is the adjoint of $A$. For given $x_{1} \in H_{1}, \lambda>0, \gamma \in\left(0, \frac{\bar{\gamma}}{\rho}\right)$, and $\epsilon \in\left(0, \frac{1}{L}\right)$, suppose that the sequences $\left\{\alpha_{n}\right\} \subset(0,1)$ and $\left\{t_{n}\right\} \subset(0, \infty)$ satisfy:

(i) $\lim _{n \rightarrow \infty} \alpha_{n}=0, \sum_{n=1}^{\infty} \alpha_{n}=\infty$, and $\sum_{n=1}^{\infty}\left|\alpha_{n}-\alpha_{n-1}\right|<\infty$;

(ii) $\lim _{n \rightarrow \infty} s_{n}=+\infty$ and $\lim _{n \rightarrow \infty} \frac{\left|s_{n}-s_{n-1}\right|}{s_{n}} \frac{1}{\alpha_{n}}=0$.

Then the sequence $\left\{x_{n}\right\}$ generated by (1.6) converges strongly to $q \in \Omega$, which is the unique solution of the following variational inequality:

$$
\langle(B-\gamma f) q, q-w\rangle \leq 0, \quad \forall w \in \Omega .
$$

Proof Taking $p \in \Omega=\operatorname{Fix}(\mathscr{T}) \cap \mathscr{Z}$, we have $p=J_{\lambda}^{B_{1}} p, A p=J_{\lambda}^{B_{2}}(A p)$, and $T(s) p=p$. From (1.6), $u_{n}=J_{\lambda}^{B_{1}}\left[x_{n}+\epsilon A^{*}\left(J_{\lambda}^{B_{2}}-I\right) A x_{n}\right]$, and Lemma 2.4, we estimate

$$
\begin{aligned}
\left\|u_{n}-p\right\|^{2} & =\left\|J_{\lambda}^{B_{1}}\left[x_{n}+\epsilon A^{*}\left(J_{\lambda}^{B_{2}}-I\right) A x_{n}\right]-J_{\lambda}^{B_{1}} p\right\|^{2} \\
& \leq\left\|x_{n}+\epsilon A^{*}\left(J_{\lambda}^{B_{2}}-I\right) A x_{n}-p\right\|^{2} \\
& \leq\left\|x_{n}-p\right\|^{2}+2 \epsilon\left\langle x_{n}-p, A^{*}\left(J_{\lambda}^{B_{2}}-I\right) A x_{n}\right\rangle+\epsilon^{2}\left\|A^{*}\left(J_{\lambda}^{B_{2}}-I\right) A x_{n}\right\|^{2} .
\end{aligned}
$$

By the definition of $A$ and $A^{*}$, we obtain

$$
\begin{aligned}
\epsilon^{2}\left\|A^{*}\left(J_{\lambda}^{B_{2}}-I\right) A x_{n}\right\|^{2} & \leq \epsilon^{2}\left\langle\left(J_{\lambda}^{B_{2}}-I\right) A x_{n}, A A^{*}\left(J_{\lambda}^{B_{2}}-I\right) A x_{n}\right\rangle \\
& \leq L \epsilon^{2}\left\langle\left(J_{\lambda}^{B_{2}}-I\right) A x_{n},\left(J_{\lambda}^{B_{2}}-I\right) A x_{n}\right\rangle \\
& =L \epsilon^{2}\left\|\left(J_{\lambda}^{B_{2}}-I\right) A x_{n}\right\|^{2} .
\end{aligned}
$$

Using a similar method to [20, Theorem 2.1] and [21, Theorem 3.1], we have

$$
\begin{aligned}
\Lambda & =2 \epsilon\left\langle x_{n}-p, A^{*}\left(J_{\lambda}^{B_{2}}-I\right) A x_{n}\right\rangle \\
& =2 \epsilon\left\langle A\left(x_{n}-p\right),\left(J_{\lambda}^{B_{2}}-I\right) A x_{n}\right\rangle
\end{aligned}
$$




$$
\begin{aligned}
& =2 \epsilon\left\langle A\left(x_{n}-p\right)+\left(J_{\lambda}^{B_{2}}-I\right) A x_{n}-\left(J_{\lambda}^{B_{2}}-I\right) A x_{n},\left(J_{\lambda}^{B_{2}}-I\right) A x_{n}\right\rangle \\
& =2 \epsilon\left[\left\langle J_{\lambda}^{B_{2}} A x_{n}-A p,\left(J_{\lambda}^{B_{2}}-I\right) A x_{n}\right\rangle-\left\|\left(J_{\lambda}^{B_{2}}-I\right) A x_{n}\right\|^{2}\right] \\
& \leq 2 \epsilon\left[\frac{1}{2}\left\|\left(J_{\lambda}^{B_{2}}-I\right) A x_{n}\right\|^{2}-\left\|\left(J_{\lambda}^{B_{2}}-I\right) A x_{n}\right\|^{2}\right] \\
& \leq-\epsilon\left\|\left(J_{\lambda}^{B_{2}}-I\right) A x_{n}\right\|^{2} .
\end{aligned}
$$

Combining (3.1) and (3.2), we obtain

$$
\left\|u_{n}-p\right\|^{2} \leq\left\|x_{n}-p\right\|^{2}+\epsilon(L \epsilon-1)\left\|\left(J_{\lambda}^{B_{2}}-I\right) A x_{n}\right\|^{2} .
$$

Setting $w_{n}=\frac{1}{s_{n}} \int_{0}^{s_{n}} T(s) u_{n} d s$ for $n \geq 0$, it follows from $\epsilon \in\left(0, \frac{1}{L}\right)$ and (3.3) that

$$
\left\|w_{n}-p\right\|=\left\|\frac{1}{s_{n}} \int_{0}^{s_{n}}\left[T(s) u_{n}-T(s) p\right] d s\right\| \leq\left\|u_{n}-p\right\| \leq\left\|x_{n}-p\right\| .
$$

It follows from (1.6), (3.4), and Lemma 2.5 that

$$
\begin{aligned}
\left\|x_{n+1}-p\right\| & =\left\|\alpha_{n}\left(\gamma f\left(x_{n}\right)-B p\right)+\left(I-\alpha_{n} B\right) \frac{1}{s_{n}} \int_{0}^{s_{n}}\left[T(s) u_{n}-T(s) p\right] d s\right\| \\
& \leq \alpha_{n}\left\|\gamma f\left(x_{n}\right)-B p\right\|+\left(1-\alpha_{n} \bar{\gamma}\right)\left\|\frac{1}{s_{n}} \int_{0}^{s_{n}}\left[T(s) u_{n}-T(s) p\right] d s\right\| \\
& \leq \alpha_{n} \gamma\left\|f\left(x_{n}\right)-f(p)\right\|+\alpha_{n}\|\gamma f(p)-B p\|+\left(1-\alpha_{n} \bar{\gamma}\right)\left\|u_{n}-p\right\| \\
& \leq\left[1-\alpha_{n}(\bar{\gamma}-\gamma \rho)\right]\left\|x_{n}-p\right\|+\alpha_{n}\|\gamma f(p)-B p\| .
\end{aligned}
$$

By a simple induction, we have

$$
\left\|x_{n}-p\right\| \leq \max \left\{\left\|x_{0}-p\right\|, \frac{1}{\bar{\gamma}-\gamma \rho}\|\gamma f(p)-B p\|\right\} .
$$

Therefore, $\left\{x_{n}\right\}$ is bounded, and so are $\left\{u_{n}\right\}$ and $\left\{w_{n}\right\}$.

Now, we show that $\lim _{n \rightarrow \infty}\left\|x_{n+1}-x_{n}\right\|=0$. From (1.6), we have

$$
\begin{aligned}
\left\|x_{n+1}-x_{n}\right\|= & \| \alpha_{n} \gamma\left[f\left(x_{n}\right)-f\left(x_{n-1}\right)\right]+\left(\alpha_{n}-\alpha_{n-1}\right) \gamma f\left(x_{n-1}\right) \\
& +\left(I-\alpha_{n} B\right)\left(w_{n}-w_{n-1}\right)-\left(\alpha_{n}-\alpha_{n-1}\right) B w_{n-1} \| \\
\leq & \alpha_{n} \gamma \rho\left\|x_{n}-x_{n-1}\right\|+\left(1-\alpha_{n} \bar{\gamma}\right)\left\|w_{n}-w_{n-1}\right\| \\
& +\left|\alpha_{n}-\alpha_{n-1}\right|\left[\left\|B w_{n-1}\right\|+\gamma\left\|f\left(x_{n-1}\right)\right\|\right] .
\end{aligned}
$$

On the other hand, for $p \in \Omega$, we have

$$
\begin{aligned}
\left\|w_{n}-w_{n-1}\right\|= & \| \frac{1}{s_{n}} \int_{0}^{s_{n}}\left[T(s) u_{n}-T(s) u_{n-1}\right] d s \\
& +\left(\frac{1}{s_{n}}-\frac{1}{s_{n-1}}\right) \int_{0}^{s_{n-1}}\left[T(s) u_{n-1}-T(s) p\right] d s \\
& +\frac{1}{s_{n}} \int_{s_{n-1}}^{s_{n}}\left[T(s) u_{n-1}-T(s) p\right] d s \| .
\end{aligned}
$$


Given that

$$
\left(\frac{1}{v}-\frac{1}{w}\right) w=-\frac{v-w}{v}, \quad v, w \neq 0
$$

It follows from (3.7) that

$$
\left\|w_{n}-w_{n-1}\right\| \leq\left\|u_{n}-u_{n-1}\right\|+\left(\frac{2\left|s_{n}-s_{n-1}\right|}{s_{n}}\right)\left\|u_{n-1}-p\right\| .
$$

Moreover, for $\epsilon \in\left(0, \frac{1}{L}\right)$, mapping $J_{\lambda}^{B_{1}}\left[I+\epsilon A^{*}\left(J_{\lambda}^{B_{2}}-I\right) A\right]$ is averaged and hence nonexpansive, then we have

$$
\begin{aligned}
\left\|u_{n}-u_{n-1}\right\| & =\left\|J_{\lambda}^{B_{1}}\left[x_{n}+\epsilon A^{*}\left(J_{\lambda}^{B_{2}}-I\right) A x_{n}\right]-J_{\lambda}^{B_{1}}\left[x_{n-1}+\epsilon A^{*}\left(J_{\lambda}^{B_{2}}-I\right) A x_{n-1}\right]\right\| \\
& \leq\left\|J_{\lambda}^{B_{1}}\left[I+\epsilon A^{*}\left(J_{\lambda}^{B_{2}}-I\right) A\right] x_{n}-J_{\lambda}^{B_{1}}\left[I+\epsilon A^{*}\left(J_{\lambda}^{B_{2}}-I\right) A\right] x_{n-1}\right\| \\
& \leq\left\|x_{n}-x_{n-1}\right\| .
\end{aligned}
$$

Combining (3.6), (3.8), and (3.9), we obtain

$$
\begin{aligned}
\left\|x_{n+1}-x_{n}\right\| \leq & \alpha_{n} \gamma \rho\left\|x_{n}-x_{n-1}\right\|+\left(1-\alpha_{n} \bar{\gamma}\right)\left[\left\|x_{n}-x_{n-1}\right\|+\left(\frac{2\left|s_{n}-s_{n-1}\right|}{s_{n}}\right)\left\|u_{n-1}-p\right\|\right] \\
& +\left|\alpha_{n}-\alpha_{n-1}\right|\left[\left\|B w_{n-1}\right\|+\gamma\left\|f\left(x_{n-1}\right)\right\|\right] \\
\leq & {\left[1-\alpha_{n}(\bar{\gamma}-\gamma \rho)\right]\left\|x_{n}-x_{n-1}\right\|+\left(\left|\alpha_{n}-\alpha_{n-1}\right|+\frac{2\left|s_{n}-s_{n-1}\right|}{s_{n}}\right) M_{1} }
\end{aligned}
$$

where $M_{1}=\max \left\{\sup _{n \in N}\left[\left\|B w_{n-1}\right\|+\gamma\left\|f\left(x_{n-1}\right)\right\|\right]\right.$, $\left.\sup _{n \in N}\left\|u_{n-1}-p\right\|\right\}$. It follows from conditions (i)-(ii) and Lemma 2.7 that

$$
\lim _{n \rightarrow \infty}\left\|x_{n+1}-x_{n}\right\|=0
$$

Next, we will show that $\lim _{n \rightarrow \infty}\left\|x_{n}-u_{n}\right\|=0$. Note that $w_{n}=\frac{1}{s_{n}} \int_{0}^{s_{n}} T(s) u_{n} d s$ and

$$
\begin{aligned}
\left\|x_{n}-w_{n}\right\| & \leq\left\|x_{n}-x_{n+1}\right\|+\left\|x_{n+1}-w_{n}\right\| \\
& =\left\|x_{n}-x_{n+1}\right\|+\left\|\alpha_{n} \gamma f\left(x_{n}\right)+\left(I-\alpha_{n} B\right) w_{n}-w_{n}\right\| \\
& \leq\left\|x_{n}-x_{n+1}\right\|+\alpha_{n}\left\|\gamma f\left(x_{n}\right)-B w_{n}\right\| .
\end{aligned}
$$

Together with condition (i) and (3.11), we obtain

$$
\lim _{n \rightarrow \infty}\left\|x_{n}-w_{n}\right\|=\lim _{n \rightarrow \infty}\left\|x_{n}-\frac{1}{s_{n}} \int_{0}^{s_{n}} T(s) u_{n} d s\right\|=0 .
$$

Observe that

$$
\begin{aligned}
\left\|x_{n}-T(u) x_{n}\right\| \leq & \left\|x_{n}-\frac{1}{s_{n}} \int_{0}^{s_{n}} T(s) u_{n} d s\right\| \\
& +\left\|\frac{1}{s_{n}} \int_{0}^{s_{n}} T(s) u_{n} d s-T(u) \frac{1}{s_{n}} \int_{0}^{s_{n}} T(s) u_{n} d s\right\|
\end{aligned}
$$




$$
\begin{aligned}
& +\left\|T(u) \frac{1}{s_{n}} \int_{0}^{s_{n}} T(s) u_{n} d s-T(u) x_{n}\right\| \\
\leq & 2\left\|x_{n}-\frac{1}{s_{n}} \int_{0}^{s_{n}} T(s) u_{n} d s\right\| \\
& +\left\|\frac{1}{s_{n}} \int_{0}^{s_{n}} T(s) u_{n} d s-T(u) \frac{1}{s_{n}} \int_{0}^{s_{n}} T(s) u_{n} d s\right\| .
\end{aligned}
$$

It follows from (3.12) and Lemma 2.2 that

$$
\lim _{n \rightarrow \infty}\left\|x_{n}-T(u) x_{n}\right\|=0 .
$$

By (3.3), (3.4), and Lemma 2.1, we have

$$
\begin{aligned}
\left\|x_{n+1}-p\right\|^{2} & =\left\|w_{n}-p+\alpha_{n}\left[\gamma f\left(x_{n}\right)-B w_{n}\right]\right\|^{2} \\
& \leq\left\|w_{n}-p\right\|^{2}+2 \alpha_{n}\left\langle\gamma f\left(x_{n}\right)-B w_{n}, x_{n+1}-p\right\rangle \\
& \leq\left\|u_{n}-p\right\|^{2}+2 \alpha_{n}\left\langle\gamma f\left(x_{n}\right)-B w_{n}, x_{n+1}-p\right\rangle \\
& \leq\left[\left\|x_{n}-p\right\|^{2}+\epsilon(L \epsilon-1)\left\|\left(J_{\lambda}^{B_{2}}-I\right) A x_{n}\right\|^{2}\right]+2 \alpha_{n}\left\langle\gamma f\left(x_{n}\right)-B w_{n}, x_{n+1}-p\right\rangle \\
& \leq\left\|x_{n}-p\right\|^{2}-\epsilon(1-L \epsilon)\left\|\left(J_{\lambda}^{B_{2}}-I\right) A x_{n}\right\|^{2}+2 \alpha_{n} M_{2}^{2},
\end{aligned}
$$

where $M_{2}=\max \left\{\sup _{n \in N}\left\|\gamma f\left(x_{n}\right)-B w_{n}\right\|, \sup _{n \in N}\left\|x_{n+1}-p\right\|\right\}$ and $\epsilon \in\left(0, \frac{1}{L}\right)$, which implies that

$$
\begin{aligned}
\epsilon(1-L \epsilon)\left\|\left(J_{\lambda}^{B_{2}}-I\right) A x_{n}\right\|^{2} & \leq\left\|x_{n}-p\right\|^{2}-\left\|x_{n+1}-p\right\|^{2}+2 \alpha_{n} M_{2}^{2} \\
& \leq\left\|x_{n+1}-x_{n}\right\|\left(\left\|x_{n}-p\right\|+\left\|x_{n+1}-p\right\|\right)+2 \alpha_{n} M_{2}^{2} .
\end{aligned}
$$

It follows from condition (i) and (3.11) that

$$
\lim _{n \rightarrow \infty}\left\|\left(J_{\lambda}^{B_{2}}-I\right) A x_{n}\right\|=0 .
$$

Furthermore, using (3.1), (3.3), and $\epsilon \in\left(0, \frac{1}{L}\right)$, we obtain

$$
\begin{aligned}
\left\|u_{n}-p\right\|^{2}= & \left\|J_{\lambda}^{B_{1}}\left[x_{n}+\epsilon A^{*}\left(J_{\lambda}^{B_{2}}-I\right) A x_{n}\right]-J_{\lambda}^{B_{1}} p\right\|^{2} \\
\leq & \left\langle u_{n}-p, x_{n}+\epsilon A^{*}\left(J_{\lambda}^{B_{2}}-I\right) A x_{n}-p\right\rangle \\
= & \frac{1}{2}\left\{\left\|u_{n}-p\right\|^{2}+\left\|x_{n}+\epsilon A^{*}\left(J_{\lambda}^{B_{2}}-I\right) A x_{n}-p\right\|^{2}\right. \\
& \left.-\left\|u_{n}-p-\left[x_{n}+\epsilon A^{*}\left(J_{\lambda}^{B_{2}}-I\right) A x_{n}-p\right]\right\|^{2}\right\} \\
\leq & \frac{1}{2}\left\{\left\|u_{n}-p\right\|^{2}+\left\|x_{n}-p\right\|^{2}+\epsilon(L \epsilon-1)\left\|\left(J_{\lambda}^{B_{2}}-I\right) A x_{n}\right\|^{2}\right. \\
& \left.-\left\|u_{n}-x_{n}-\epsilon A^{*}\left(J_{\lambda}^{B_{2}}-I\right) A x_{n}\right\|^{2}\right\} \\
\leq & \frac{1}{2}\left\{\left\|u_{n}-p\right\|^{2}+\left\|x_{n}-p\right\|^{2}-\left[\left\|u_{n}-x_{n}\right\|^{2}+\epsilon^{2}\left\|A^{*}\left(J_{\lambda}^{B_{2}}-I\right) A x_{n}\right\|^{2}\right.\right. \\
& \left.\left.-2 \epsilon\left\langle u_{n}-x_{n}, A^{*}\left(J_{\lambda}^{B_{2}}-I\right) A x_{n}\right)\right]\right\} \\
\leq & \frac{1}{2}\left\{\left\|u_{n}-p\right\|^{2}+\left\|x_{n}-p\right\|^{2}-\left\|u_{n}-x_{n}\right\|^{2}+2 \epsilon\left\|A\left(u_{n}-x_{n}\right)\right\|\left\|\left(J_{\lambda}^{B_{2}}-I\right) A x_{n}\right\|\right\}
\end{aligned}
$$


which implies that

$$
\left\|u_{n}-p\right\|^{2} \leq\left\|x_{n}-p\right\|^{2}-\left\|u_{n}-x_{n}\right\|^{2}+2 \epsilon\left\|A\left(u_{n}-x_{n}\right)\right\|\left\|\left(J_{\lambda}^{B_{2}}-I\right) A x_{n}\right\| .
$$

It follows from (3.14) and (3.17) that

$$
\begin{aligned}
\left\|x_{n+1}-p\right\|^{2} & \leq\left\|u_{n}-p\right\|^{2}+2 \alpha_{n} M_{2}^{2} \\
& \leq\left\|x_{n}-p\right\|^{2}-\left\|u_{n}-x_{n}\right\|^{2}+2 \epsilon\left\|A\left(u_{n}-x_{n}\right)\right\|\left\|\left(J_{\lambda}^{B_{2}}-I\right) A x_{n}\right\|+2 \alpha_{n} M_{2}^{2},
\end{aligned}
$$

that is,

$$
\begin{aligned}
\left\|u_{n}-x_{n}\right\|^{2} \leq & \left\|x_{n}-p\right\|^{2}-\left\|x_{n+1}-p\right\|^{2}+2 \epsilon\left\|A\left(u_{n}-x_{n}\right)\right\|\left\|\left(J_{\lambda}^{B_{2}}-I\right) A x_{n}\right\|+2 \alpha_{n} M_{2}^{2} \\
\leq & \left\|x_{n}-x_{n+1}\right\|\left(\left\|x_{n}-p\right\|+\left\|x_{n+1}-p\right\|\right) \\
& +2 \epsilon\left\|A\left(u_{n}-x_{n}\right)\right\|\left\|\left(J_{\lambda}^{B_{2}}-I\right) A x_{n}\right\|+2 \alpha_{n} M_{2}^{2} .
\end{aligned}
$$

Combining condition (i), (3.11), and (3.16), we have

$$
\lim _{n \rightarrow \infty}\left\|u_{n}-x_{n}\right\|=0 .
$$

Since $\left\{x_{n}\right\}$ and $\left\{u_{n}\right\}$ are bounded, we consider a weak cluster point $w$ of $\left\{x_{n}\right\}$. Without loss of generality, we may assume that subsequence $\left\{x_{n_{j}}\right\}$ of $\left\{x_{n}\right\}$ converges weakly to $w$, i.e., $x_{n_{j}} \rightarrow w$ as $j \rightarrow \infty$. From (3.18), we have $\left\{u_{n_{j}}\right\}$ of $\left\{u_{n}\right\}$, which converges weakly to $w$. Moreover, $u_{n_{j}}=J_{\lambda}^{B_{1}}\left[x_{n_{j}}+\epsilon A^{*}\left(J_{\lambda}^{B_{2}}-I\right) A x_{n_{j}}\right]$ can be rewritten as

$$
\frac{\left(x_{n_{j}}-u_{n_{j}}\right)+\epsilon A^{*}\left(J_{\lambda}^{B_{2}}-I\right) A x_{n_{j}}}{\lambda} \in B_{1} u_{n_{j}} .
$$

By passing to the limit $j \rightarrow \infty$ in (3.19) and by taking into account (3.16), (3.18), and the fact that the graph of a maximal monotone operator is weakly-strongly closed, we obtain $0 \in B_{1}(w)$. Furthermore, since $\left\{x_{n}\right\}$ and $\left\{u_{n}\right\}$ have the same asymptotical behavior, $\left\{A x_{n_{j}}\right\}$ weakly converges to $A w$. From (3.16) and the fact that the resolvent $J_{\lambda}^{B_{2}}$ is nonexpansive, we obtain $A w \in B_{2}(A w)$. It follows from Lemma 2.4 that $w \in \mathscr{Z}$.

We now show that $\limsup _{n \rightarrow \infty}\left\langle\gamma f(q)-B q, x_{n}-q\right\rangle \leq 0$, where $q=P_{\Omega}(I-B+\gamma f) q$. Note that the subsequence $\left\{x_{n_{j}}\right\}$ of $\left\{x_{n}\right\}$ converges weakly to $w$ and

$$
\limsup _{n \rightarrow \infty}\left\langle\gamma f(q)-B q, x_{n}-q\right\rangle=\lim _{j \rightarrow \infty}\left\langle\gamma f(q)-B q, x_{n_{j}}-q\right\rangle .
$$

Assume that $w \neq T(u) w$. By (3.13) and Opial's property, we obtain

$$
\begin{aligned}
\liminf _{j \rightarrow \infty}\left\|x_{n_{j}}-w\right\| & <\liminf _{j \rightarrow \infty}\left\|x_{n_{j}}-T(u) w\right\| \\
& \leq \liminf _{j \rightarrow \infty}\left(\left\|x_{n_{j}}-T(u) x_{n_{j}}\right\|+\left\|T(u) x_{n_{j}}-T(u) w\right\|\right) \\
& \leq \liminf _{j \rightarrow \infty}\left(\left\|x_{n_{j}}-T(u) x_{n_{j}}\right\|+\left\|x_{n_{j}}-w\right\|\right) \\
& \leq \liminf _{j \rightarrow \infty}\left\|x_{n_{j}}-w\right\| .
\end{aligned}
$$


This is a contradiction. Then $w \in \operatorname{Fix}(\mathscr{T})$. Consequently, $w \in \Omega=\operatorname{Fix}(\mathscr{T}) \cap \mathscr{Z}$. It follows from (3.20) that

$$
\limsup _{n \rightarrow \infty}\left\langle\gamma f(q)-B q, x_{n}-q\right\rangle=\langle\gamma f(q)-B q, w-q\rangle \leq 0 .
$$

On the other hand, we shall show that the uniqueness of a solution of the variational inequality

$$
\langle(B-\gamma f) x, x-w\rangle \leq 0, \quad w \in \Omega .
$$

Suppose $q \in \Omega$ and $\hat{q} \in \Omega$ both are solutions to (3.22), then

$$
\langle(B-\gamma f) q, q-\hat{q}\rangle \leq 0
$$

and

$$
\langle(B-\gamma f) \hat{q}, \hat{q}-q\rangle \leq 0
$$

Adding up (3.23) and (3.24) one gets

$$
\langle(B-\gamma f) q-(B-\gamma f) \hat{q}, q-\hat{q}\rangle \leq 0 .
$$

By Lemma 2.6, the strong monotonicity of $B-\gamma f$, we obtain $q=\hat{q}$ and the uniqueness is proved.

Finally, we show that $\left\{x_{n}\right\}$ converges strongly to $q$ as $n \rightarrow \infty$. From (1.6), (3.4), and Lemma 2.1, we have (note that $w_{n}=\frac{1}{s_{n}} \int_{0}^{s_{n}} T(s) u_{n} d s$ )

$$
\begin{aligned}
\left\|x_{n+1}-q\right\|^{2}= & \left\langle\alpha_{n} \gamma f\left(x_{n}\right)+\left(I-\alpha_{n} B\right) w_{n}-q, x_{n+1}-q\right\rangle \\
= & \alpha_{n}\left\langle\gamma f\left(x_{n}\right)-B q, x_{n+1}-q\right\rangle+\left\langle\left(I-\alpha_{n} B\right)\left(w_{n}-q\right), x_{n+1}-q\right\rangle \\
\leq & \alpha_{n} \gamma\left\langle f\left(x_{n}\right)-f(q), x_{n+1}-q\right\rangle+\alpha_{n}\left\langle\gamma f(q)-B q, x_{n+1}-q\right\rangle \\
& +\left(1-\alpha_{n} \bar{\gamma}\right)\left\|w_{n}-q\right\|\left\|x_{n+1}-q\right\| \\
\leq & \alpha_{n} \gamma \rho\left\|x_{n}-q\right\|\left\|x_{n+1}-q\right\|+\alpha_{n}\left\langle\gamma f(q)-B q, x_{n+1}-q\right\rangle \\
& +\left(1-\alpha_{n} \bar{\gamma}\right)\left\|x_{n}-q\right\|\left\|x_{n+1}-q\right\| \\
= & {\left[1-\alpha_{n}(\bar{\gamma}-\gamma \rho)\right]\left\|x_{n}-q\right\|\left\|x_{n+1}-q\right\|+\alpha_{n}\left\langle\gamma f(q)-B q, x_{n+1}-q\right\rangle } \\
\leq & \frac{1-\alpha_{n}(\bar{\gamma}-\gamma \rho)}{2}\left(\left\|x_{n}-q\right\|^{2}+\left\|x_{n+1}-q\right\|^{2}\right)+\alpha_{n}\left\langle\gamma f(q)-B q, x_{n+1}-q\right\rangle \\
\leq & \frac{1-\alpha_{n}(\bar{\gamma}-\gamma \rho)}{2}\left\|x_{n}-q\right\|^{2}+\frac{1}{2}\left\|x_{n+1}-q\right\|^{2}+\alpha_{n}\left\langle\gamma f(q)-B q, x_{n+1}-q\right\rangle .
\end{aligned}
$$

It follows that

$$
\left\|x_{n+1}-q\right\|^{2} \leq\left[1-(\bar{\gamma}-\gamma \rho) \alpha_{n}\right]\left\|x_{n}-q\right\|^{2}+2 \alpha_{n}\left\langle\gamma f(q)-B q, x_{n+1}-q\right\rangle .
$$

From $0<\gamma<\frac{\bar{\gamma}}{\rho}$, condition (i), and (3.21), we can arrive at the desired conclusion $\lim _{n \rightarrow \infty}\left\|x_{n}-q\right\|=0$ by applying Lemma 2.7 to (3.26). This completes the proof. 
Theorem 3.3 Let $H_{1}$ and $H_{2}$ be two real Hilbert spaces and $A: H_{1} \rightarrow H_{2}$ be a bounded linear operator. Let $B_{1}: H_{1} \rightarrow 2^{H_{1}}, B_{2}: H_{2} \rightarrow 2^{H_{1}}$ be maximal monotone mappings and $\mathscr{T}:=\{T(s): 0 \leq s<\infty\}$ be a one-parameter nonexpansive semigroup on $H_{1}$ such that $\Omega=$ $\operatorname{Fix}(\mathscr{T}) \cap \mathscr{Z} \neq \emptyset$. Assume that $f: H_{1} \rightarrow H_{1}$ is a contraction mapping with constant $\rho \in$ $(0,1), L$ is the spectral radius of the operator $A^{*} A$, and $A^{*}$ is the adjoint of $A$. For given $x_{1} \in H_{1}, \lambda>0$, and $\epsilon \in\left(0, \frac{1}{L}\right)$, define $\left\{x_{n}\right\}$ in the following manner:

$$
x_{n+1}=\alpha_{n} f\left(x_{n}\right)+\left(1-\alpha_{n}\right) \frac{1}{s_{n}} \int_{0}^{s_{n}} T(s) J_{\lambda}^{B_{1}}\left[x_{n}+\epsilon A^{*}\left(J_{\lambda}^{B_{2}}-I\right) A x_{n}\right] d s
$$

where the sequence $\left\{\alpha_{n}\right\} \subset(0,1)$ satisfies the following conditions:

(i) $\lim _{n \rightarrow \infty} \alpha_{n}=0, \sum_{n=1}^{\infty} \alpha_{n}=\infty$, and $\sum_{n=1}^{\infty}\left|\alpha_{n}-\alpha_{n-1}\right|<\infty$;

(ii) $\lim _{n \rightarrow \infty} s_{n}=+\infty$ and $\lim _{n \rightarrow \infty} \frac{\left|s_{n}-s_{n-1}\right|}{s_{n}} \frac{1}{\alpha_{n}}=0$.

Then the sequence $\left\{x_{n}\right\}$ converges strongly to $q=P_{\Omega} f(q)$, which is equivalent to the unique solution of the following variational inequality:

$$
\langle(I-f) q, q-w\rangle \leq 0, \quad \forall w \in \Omega
$$

Proof Putting $\gamma=1$ and $B=I$, iterative scheme (1.6) reduces to viscosity iteration (3.27). The desired conclusion follows immediately from Theorem 3.2. This completes the proof.

Theorem 3.4 Let $H_{1}$ and $H_{2}$ be two real Hilbert spaces, let $A: H_{1} \rightarrow H_{2}$ be a bounded linear operator and $B$ be a strongly positive bounded linear operator on $H_{1}$ with constant $\bar{\gamma}>0$. Let $B_{1}: H_{1} \rightarrow 2^{H_{1}}, B_{2}: H_{2} \rightarrow 2^{H_{1}}$ be maximal monotone mappings and $T: H_{1} \rightarrow H_{1}$ be a nonexpansive mapping such that $\Omega=\operatorname{Fix}(T) \cap \mathscr{Z} \neq \emptyset$. Assume that $f: H_{1} \rightarrow H_{1}$ is a contraction mapping with constant $\rho \in(0,1), L$ is the spectral radius of the operator $A^{*} A$, and $A^{*}$ is the adjoint of $A$. For given $x_{1} \in H_{1}, \lambda>0, \gamma \in\left(0, \frac{\bar{\gamma}}{\rho}\right)$, and $\epsilon \in\left(0, \frac{1}{L}\right)$, define $\left\{x_{n}\right\}$ in the following manner:

$$
\left\{\begin{array}{l}
u_{n}=J_{\lambda}^{B_{1}}\left[x_{n}+\epsilon A^{*}\left(J_{\lambda}^{B_{2}}-I\right) A x_{n}\right], \\
x_{n+1}=\alpha_{n} \gamma f\left(x_{n}\right)+\left(I-\alpha_{n} B\right) T u_{n},
\end{array}\right.
$$

where the sequence $\left\{\alpha_{n}\right\} \subset(0,1)$ satisfies $\lim _{n \rightarrow \infty} \alpha_{n}=0, \sum_{n=1}^{\infty} \alpha_{n}=\infty$, and $\sum_{n=1}^{\infty} \mid \alpha_{n}-$ $\alpha_{n-1} \mid<\infty$. Then the sequence $\left\{x_{n}\right\}$ converges strongly to $q$, which is the unique solution of the following variational inequality:

$$
\langle(B-\gamma f) q, q-w\rangle \leq 0, \quad \forall w \in \Omega
$$

Proof Clearly, Theorem 3.2 is valid for a nonexpansive mapping. Therefore, the desired conclusion follows immediately from Theorem 3.2. This completes the proof.

Remark 3.1 Theorems 3.2 and 3.3 extend the approximation scheme of Byrne et al. [15] and the viscosity results of Kazmi and Rizvi [16] to a general iterative method for a split variational inclusion and one-parameter nonexpansive semigroups, which includes the results of $[15,16]$ as special cases. 
Table 1 Numerical results for some initial points $x_{1}=-1,0,1,2,15$

\begin{tabular}{clllll}
\hline Iter. $(\boldsymbol{n})$ & $\boldsymbol{x}_{\boldsymbol{n}}^{(\mathbf{1})}$ & $\boldsymbol{x}_{\boldsymbol{n}}^{(\mathbf{2})}$ & $\boldsymbol{x}_{\boldsymbol{n}}^{(\mathbf{3})}$ & $\boldsymbol{x}_{\boldsymbol{n}}^{(\mathbf{4})}$ & $\boldsymbol{x}_{\boldsymbol{n}}^{(\mathbf{5})}$ \\
\hline 0 & -1.0000 & 0.0000 & 1.0000 & 2.0000 & 15.000 \\
1 & -0.5000 & 0.0000 & 0.5000 & 1.0000 & 7.5000 \\
2 & -0.1891 & 0.0000 & 0.1891 & 0.3781 & 2.8358 \\
3 & -0.0600 & 0.0000 & 0.6000 & 0.1199 & 0.8996 \\
4 & -0.0167 & 0.0000 & 0.0167 & 0.0334 & 0.2506 \\
5 & -0.0042 & 0.0000 & 0.0042 & 0.0084 & 0.0630 \\
$\ldots$ & $\ldots$ & $\ldots$ & $\ldots$ & $\ldots$ & $\ldots$ \\
8 & 0.0000 & 0.0000 & 0.0000 & 0.0001 & 0.0006 \\
9 & 0.0000 & 0.0000 & 0.0000 & 0.0000 & 0.0001 \\
10 & 0.0000 & 0.0000 & 0.0000 & 0.0000 & 0.0000 \\
\hline
\end{tabular}

Remark 3.2 Theorems 3.2 and 3.4 improve and extend the main results of Marino and $\mathrm{Xu}$ [2] for nonexpansive mappings, and [3-5] for nonexpansive semigroups in different directions.

\section{Numerical results}

In this section, we give an example and numerical results to illustrate our algorithm and the main result of this paper.

Example 4.1 Let $H_{1}=H_{2}=\mathbb{R}$. Let $B_{1} x=2 x$ and $B_{2} x=3 x$. Let $\mathscr{T}:=\{T(s): 0 \leq s<\infty\}$, where $T(s) x=\frac{1}{1+2 s} x, \forall x \in \mathbb{R}$. Then $B_{1}, B_{2}$, and $\mathscr{T}$ satisfy all conditions of Theorem 3.2 and $\Omega=\operatorname{Fix}(\mathscr{T}) \cap \mathscr{L}=\{0\}$. Let $\left\{x_{n}\right\}$ be the sequence generated by $x_{0}$ and

$$
x_{n+1}=\alpha_{n} \gamma f\left(x_{n}\right)+\left(I-\alpha_{n} B\right) \frac{1}{s_{n}} \int_{0}^{s_{n}} \frac{1}{1+2 s} J_{\lambda}^{B_{1}}\left[x_{n}+\epsilon A^{*}\left(J_{\lambda}^{B_{2}}-I\right) A x_{n}\right] d s .
$$

Let $A=B=I$, the identity operator, and $f(x)=\frac{1}{2} x, \forall x \in \mathbb{R}$. Putting $\gamma=\lambda=1, \epsilon=\frac{1}{2}$, and $\alpha_{n}=\frac{1}{\sqrt{n}}, s_{n}=n$, then scheme (4.1) reduces to

$$
x_{n+1}=\frac{1}{2 \sqrt{n}}\left[x_{n}+\frac{5}{24 n}(\sqrt{n}-1) \ln (1+2 n) x_{n}\right] \text {. }
$$

Setting $\left\|x_{n}-x^{*}\right\| \leq 10^{-4}$ as stop criterion, then we obtain the numerical results of scheme (4.2) with different initial points $x_{1}$ in Table 1.

The computations are performed by Matlab R2007a running on a PC Desktop Intel(R) Core(TM)i3-2330M, CPU @2.20 GHz, 790 MHz, 1.83 GB, 2 GB RAM.

Competing interests
The authors declare that they have no competing interests.

\section{Authors' contributions}

D-JW carried out the primary studies of a split variational inclusion and the fixed point problem of nonexpansive semigroups and drafted the manuscript. Y-AC participated in algorithm design and convergence analysis. All authors read and approved the final manuscript.

\section{Acknowledgements}

The authors are grateful to the anonymous editor and referees for valuable remarks suggestions, which helped them very much in improving this manuscript. This work was supported by the National Science Foundation of China (11471059), Basic and Advanced Research Project of Chongqing (cstc2014jcyjA00037), Science and Technology Research Project of Chongqing Municipal Education Commission (KJ1400614, KJ1400618). 


\section{References}

1. Browder, FE: Convergence of approximants to fixed points of nonexpansive nonlinear mappings in Banach spaces. Arch. Ration. Mech. Anal. 24, 82-89 (1967)

2. Marino, G, Xu, HK: A general iterative method for nonexpansive mappings in Hilbert spaces. J. Math. Anal. Appl. 318 43-52 (2006)

3. Li, S, Li, L, Su, Y: General iterative methods for a one-parameter nonexpansive semigroup in Hilbert space. Nonlinear Anal. 70, 3065-3071 (2009)

4. Cianciaruso, F, Marino, G, Muglia, L: Iterative methods for equilibrium and fixed point problems for nonexpansive semigroups in Hilbert spaces. J. Optim. Theory Appl. 146, 491-509 (2010)

5. Shehu, Y: An iterative method for nonexpansive semigroups, variational inclusions and generalized equilibrium problems. Math. Comput. Model. 55, 1301-1314 (2012)

6. Qin, X, Cho, SY, Kang, SM: Iterative algorithm for variational inequality and equilibrium problems with applications. J. Glob. Optim. 48, 423-445 (2010)

7. Wen, D-J, Chen, Y-A: Strong convergence of modified general iterative method for generalized equilibrium problems and fixed point problems of k-strict pseudo-contractions. Fixed Point Theory Appl. 2012, 125 (2012)

8. Yang, L: The general iterative scheme for semigroups of nonexpansive mappings and variational inequalities with applications. Math. Comput. Model. 57, 1289-1297 (2013)

9. Moudafi, A: Split monotone variational inclusions. J. Optim. Theory Appl. 150, 275-283 (2011)

10. Censor, Y, Elfving, T: A multiprojection algorithm using Bregman projections in product space. Numer. Algorithms 8 221-239 (1994)

11. Censor, Y, Bortfeld, T, Martin, B, Trofimov, A: A unified approach for inversion problems in intensity modulated radiation therapy. Phys. Med. Biol. 51, 2353-2365 (2006)

12. Censor, Y, Gibali, A, Reich, S: Algorithms for the split variational inequality problem. Numer. Algorithms 59, $301-323$ (2012)

13. Combettes, PL: The convex feasibility problem in image recovery. Adv. Imaging Electron Phys. 95, 155-453 (1996)

14. Byrne, C: Iterative oblique projection onto convex sets and the split feasibility problem. Inverse Probl. 18, 441-453 (2002)

15. Byrne, C, Censor, Y, Gibali, A, Reich, S: Weak and strong convergence of algorithms for the split common null point problem. J. Nonlinear Convex Anal. 13, 759-775 (2012)

16. Kazmi, KR, Rizvi, SH: An iterative method for split variational inclusion problem and fixed point problem for a nonexpansive mapping. Optim. Lett. (2013). doi:10.1007/s11590-013-0629-2

17. Shimizu, T, Takahashi, W: Strong convergence of common fixed points of families of nonexpansive mappings. J. Math Anal. Appl. 211, 71-83 (1997)

18. Bauschke, HH, Combettes, PL: Convex Analysis and Monotone Operator Theory in Hilbert Spaces. Springer, New York (2011)

19. Xu, HK: Iterative algorithm for nonlinear operators. J. Lond. Math. Soc. 66(2), 1-17 (2002)

20. Crombez, G: A geometrical look at iterative methods for operators with fixed points. Numer. Funct. Anal. Optim. 26 157-175 (2005)

21. Crombez, G: A hierarchical presentation of operators with fixed points on Hilbert spaces. Numer. Funct. Anal. Optim 27, 259-277 (2006)

\section{Submit your manuscript to a SpringerOpen ${ }^{\circ}$ journal and benefit from:}

- Convenient online submission

- Rigorous peer review

- Immediate publication on acceptance

- Open access: articles freely available online

- High visibility within the field

- Retaining the copyright to your article 